\title{
Detection and Clinical Value of Circulating Tumor Cells as an Assisted Prognostic Marker in Colorectal Cancer Patients
}

\author{
Rui-Jun Pan ${ }^{1-3}$ \\ Hi-Ju Hong ${ }^{1,2}$ \\ Jing Sun ${ }^{1,2}$ \\ Chao-Ran $\mathrm{Yu}^{1,2}$ \\ Hai-Shan Liu ${ }^{1,2}$ \\ Pei-Yong $\mathrm{Li}^{3} *$ \\ Min-Hua Zheng ${ }^{1,2, *}$ \\ 'Department of General Surgery, Ruijin \\ Hospital, Shanghai Jiao Tong University \\ School of Medicine, Shanghai, 200025, \\ People's Republic of China; ${ }^{2}$ Shanghai \\ Minimally Invasive Surgery Center, Ruijin \\ Hospital, Shanghai Jiao Tong University \\ School of Medicine, Shanghai, 200025, \\ People's Republic of China; ${ }^{3}$ Department \\ of Nuclear Medicine, Ruijin Hospital, \\ Shanghai Jiao Tong University School of \\ Medicine, Shanghai, 200025, People's \\ Republic of China \\ *These authors contributed equally to \\ this work
}

Correspondence: Pei-Yong Li Department of Nuclear Medicine, Ruijin Hospital, Shanghai Jiao Tong University School of Medicine, No. 197, Ruijin Second Road, Shanghai, 200025, People's Republic of China

Email peiyli@vip.sina.com

Min-Hua Zheng

Department of General Surgery, Ruijin Hospital, Shanghai Jiao Tong University School of Medicine, No. 197, Ruijin

Second Road, Shanghai, 200025, People's Republic of China

Email zmhtiger@yeah.net
Background: Circulating tumor cells (CTCs) are cells that have been shed into the vasculature from a primary tumor and circulate in the bloodstream. It has been suggested that detecting CTCs could help the clinician to detect early metastasis or recurrence more effectively. This trial sets out to assess the detection and clinical value of CTCs as an assisted prognostic marker in patients with colon cancer and rectal cancer.

Methods: A prospective cohort of patients with colorectal cancer (CRC) was enrolled from July 2015 to February 2018 in Shanghai Minimally Invasive Surgery Center, Shanghai, China. In this study, 149 patients with CRC were enrolled and underwent surgical treatment. There were 79 cases of colon cancer and 70 cases of rectal cancer, including 93 males and 56 females. To investigate the correlativity and clinical value of CTCs, the patients were statistically analyzed in different subgroups: colon cancer group vs rectal cancer group, and left hemicolon cancer group vs right hemicolon cancer group. Results: The results of analysis comparing CTC counts and clinical pathological features in colon and rectal cancer indicated that with increased tumor stage, the number of CTCs also increased, with significant statistical differences. CTC counts in patients with colon and rectal cancer showed positive correlations with TNM staging $(P=0.001,0.013$, respectively), $\mathrm{T}$ staging $(P=0.021,0.001), \mathrm{N}$ staging $(P=0.014,0.035)$ and $\mathrm{M}$ staging $(P=0.018,0.203)$. Detection of serum biomarkers in CTC-positive and CTC-negative groups indicated a significantly increasing expression in the CTC-positive group. To confirm the correlations between CTCs and histoembryological differences, analysis was conducted with the patients in two subgroups: left hemicolon cancer group and right hemicolon cancer group. The results showed that the positive rate of CTCs increased in both groups with the increase in tumor stage. The survival analysis indicated that there was a steep gradient in survival in the follow-up period, particularly in the CTC-positive group $(P=0.000)$. Risk assessment curves showed that the change escalated more rapidly in the CTC-positive group. Furthermore, with the increase in $\mathrm{T}$ stage, changes in the survival curve and risk curve escalated more rapidly in the CTCpositive group.

Conclusion: It was confirmed that in the left hemicolon cancer group, a much higher coincidence rate could be found on CTC-positive rate and clinicopathological features, than in the right hemicolon cancer group. The sensitivity of CTCs may be related to the histoembryological location of the tumor, lymphatic metastasis and the depth of infiltration. Monitoring CTCs may have value in evaluating clinical staging and estimating clinical prognosis.

Keywords: CTC, colorectal cancer, prognostic marker, clinical value 


\section{Introduction}

Colorectal cancer (CRC) is one of the most common malignant tumors in the digestive system. With the westernization of the diet, China is witnessing rising rates of morbidity and mortality due to CRC. ${ }^{1}$ Surgical treatment combined with radiochemotherapy is considered the most effective chemotherapeutic treatment of CRC according to the guidelines of the National Comprehensive Cancer Network (NCCN), European Society for Medical Oncology (ESMO) Consensus Guidelines and Chinese Society of Clinical Oncology (CSCO). ${ }^{2-4}$ This treatment strategy shows good curative effect in cases with advanced stage tumors.

However, in recent years, with the popularization of community-based cancer screening programs, the diagnostic rate of early CRC has increased, but it is still lower than in some developed countries, such as Japan and European countries. ${ }^{1,5}$ With the popularization of colonoscopy, the rate of early diagnosis of CRC has increased greatly. However, we cannot deny that most people are usually reluctant to undergo invasive examinations, especially in China. Several tumor markers, for example, CEA, CA125, CA199 and CA724, are included in community-based cancer screening programs. ${ }^{6-8}$ It has been reported, however, that not all cases with CRC have elevated tumor indices. This is because the specificity and sensitivity of these tumor markers are not high enough for the precise detection of $\mathrm{CRC}$, leading to low rates of detection and diagnosis. Circulating tumor cells (CTCs) are cells that have been shed into the vasculature from a primary tumor and circulate in the bloodstream, and it has been suggested that detecting CTCs would help clinicians to detect early metastasis or recurrence more effectively. It has been reported that metastasis of early gastric cancer is caused by CTCs, ${ }^{9}$ and researchers have reported positive rates of CTC in patients with gastric cancer of $44.8 \%, 25 \%, 57.4 \%$ and $70 \%$ in stages I, II, III and IV. ${ }^{10,11}$ In the case of colon cancer, the follow-up results showed that the positive rate of CTCs in the postoperative 24 hours is closely related to the overall survival (OS) and disease-free survival (DFS). These results show that CTCs could be an effective prognostic marker for early diagnosis, outcome evaluation and guidance for comprehensive treatment. However, researchcorrelativity between CTCs in CRC and clinicopathological features are still lacking, especially in Chinese populations.
Therefore, in order to enable a precise diagnosis of $\mathrm{CRC}$ and predict the high-risk patients more effectively, ${ }^{12-14}$ a cohort study was performed in the patients with $\mathrm{CRC}$ in our center to enable the clinical evaluation of CTCs. ${ }^{15,16}$

\section{Methods}

\section{Patient Enrollment}

A prospective cohort of CRC patients was enrolled from July 2015 to February 2018 in Shanghai Minimally Invasive Surgery Center, Shanghai, China, for this study.

The study was approved by the institutional research ethics committee of Shanghai Ruijin Hospital, and all the patients agreed and signed the medical informed consent form. This study was conducted in accordance with the Declaration of Helsinki.

\section{Inclusion Criteria}

1) Age $\geq 18$ years; 2) pathological diagnosis of CRC by colonoscopic biopsy; 3) clinical or radiological evidence of stage I-III disease (according to the 2016 revision of the International Union Against Cancer primary tumor, regional nodes, metastasis [TNM] staging system), and stage IV in CRC patients; ESMO group 0 cases were enrolled after clinical evaluation for clearly resectable liver and/or lung disease; ${ }^{17,18} 3$ ) non-emergency operation; 4) no neoadjuvant chemotherapy; 5) absence of multiple primary tumors; 6) signed medical informed consent.

\section{Exclusion Criteria}

1) Emergency operation (perforation, hemorrhage, complete obstruction); 2) ESMO group 1-3 stage IV patients; ${ }^{17,18} 3$ ) suffered from a serious medical condition (heart, lung, liver and kidney disease) and could not tolerate laparoscopic surgery; 4) rejection of formal postoperative treatment; 5) refused informed consent.

\section{Exit Criteria}

1) Participants quit the study voluntarily for various reasons; 2) participants, principal investigator, ethics committee, project leader or governor of the state food and administration consider terminating the study because of serious related adverse events; 3 ) clinicians consider terminating the study from a medical standpoint; 4) sponsors consider terminating the study because the investigator has not executed the trial; 5) participants deviate from the protocol; 6) participants do not fully or properly 
understand the information related to the trial; 7) other specific reasons require termination of the trial.

\section{Treatment and Follow-Up}

In this study, all patients with colon cancer and rectal cancer had undergone surgical treatment in Shanghai Minimally Invasive Surgery Center. Among them, stage III and highrisk stage II patients (ie, with incomplete obstruction, perforation, positive surgical margin, vascular cancer embolus, nerve infiltration, harvested lymph-node counts less than 12 and poorly differentiated carcinoma) accepted adjuvant chemotherapy (mFolfox6 or CapeOX) for 6 months. Stage IV patients and patients diagnosed with PD during the followup were arranged to undergo a tumor board discussion and an individualized treatment strategy was determined according to CSCO guidelines. Participants were followed up until death or July 30th, 2018.

\section{Detection of CTCs}

The detailed protocols for CTC detection were performed as previously described. ${ }^{17}$ In brief, blood samples were harvested preoperatively for CTC detection. The samples were centrifuged $(1500 \mathrm{rpm})$, then the upper clear supernatant was discarded and incubated with $30 \mu \mathrm{L}$ of immuno nano-magnetic spheres for 30 minutes (Magnetic nanoparticles, ShengNa Industrial Limited Company, Shanghai, China; BaiHuiKang Biotechnology Co. Shanghai, China). Cells enriched by magnetic isolation were stained by adding $2 \mu \mathrm{L}$ of diamidinophenylindole(DAPI), $10 \mu \mathrm{L}$ of CK-19-FITC and $10 \mu \mathrm{L}$ of CD45 (DAPI, BiYunTian Biotechnology Co., China; Anti-CK-19, phycoerythrin (PE), Johnson \& Johnson Medical Veridex Company, USA; Anti-CD-45-PE, Becton, Dickinson and Company, USA). Observation of CTCs was carried out after air-drying; the pictures with fluorescence staining were captured under a fluorescence microscope (Olympus B*61, USA) for the identification and counting of CTCs.

\section{Typical Criteria of CTCs}

The results with lower counts $(\leq 3$ per $7.5 \mathrm{~mL}$ of peripheral blood) of CTCs or no CTCs found in peripheral blood were defined as the negative group (CTC-).

The results with high-level counts $(>3$ per $7.5 \mathrm{~mL}$ of peripheral blood) of CTCs, which means there may be a higher risk of recurrence and metastasis, were defined as the positive group $(\mathrm{CTC}+)$.

\section{Statistical Methods}

SPSS 17.0 software was used for data analysis. Enumeration data were compared by using independent sample KruskalWallis identification exact probability method. Statistical significance was considered with $P<0.05$. Kaplan-Meier estimation was used to describe the survival function and the risk function.

\section{Results}

\section{Patient Demographic Data}

In this study, 149 patients with CRC were enrolled and underwent surgical treatment in Shanghai Minimally Invasive Surgery Center from July 2015 to December 2018 (Table 1). No patients were lost during the follow-up. The average follow-up time was $23.59 \pm 5.18$ months. There were 79 cases of colon cancer and 70 cases of rectal cancer, including 93 males and 56 females. The average age was $63.19 \pm 11.80$ years and the average body mass index (BMI) was $22.61 \pm 3.38 \mathrm{~kg} / \mathrm{m}^{2}$. In the colon cancer group, the average age was $63.38 \pm 11.74$ years and the average BMI was $22.58 \pm 3.50 \mathrm{~kg} / \mathrm{m}^{2}$; in the rectal cancer group, the average age was $62.97 \pm 11.94$ years and the average BMI was 22.64 $\pm 3.26 \mathrm{~kg} / \mathrm{m}^{2}$. According to the UICC/AJCC TNM classification, there were 42 cases with stage I, 35 cases with stage II, 58 cases with stage III and 14 cases with stage IV disease. In the colon cancer group, there were 23 cases with stage I, 20 cases with stage II, 26 cases with stage III and 10 cases with stage IV; in the rectal cancer group, there were 19 cases with stage I, 15 cases with stage II, 32 cases with stage III and four cases with stage IV. The results showed that the

Table I Preoperative Demographic Information and Pathological Features

\begin{tabular}{|l|c|c|c|}
\hline & $\begin{array}{c}\text { Total } \\
(\mathbf{n = 1 4 9 )}\end{array}$ & $\begin{array}{c}\text { Colon } \\
\text { Cancer } \\
(\mathbf{n = 7 9 )}\end{array}$ & $\begin{array}{c}\text { Rectal } \\
\text { Cancer } \\
(\mathbf{n}=70)\end{array}$ \\
\hline Age (years) & $63.19 \pm 11.80$ & $63.38 \pm 11.74$ & $62.97 \pm 11.94$ \\
Gender (M/F) & $93 / 56$ & $46 / 33$ & $47 / 23$ \\
BMI $\left(\mathrm{kg} / \mathrm{m}^{2}\right)$ & $22.61 \pm 3.38$ & $22.58 \pm 3.50$ & $22.64 \pm 3.26$ \\
\hline Stage I & 42 & 23 & 19 \\
Stage II & 35 & 20 & 15 \\
Stage III & 58 & 26 & 32 \\
Stage IV & 14 & 10 & 4 \\
\hline CEA (ng/mL) & $13.33 \pm 33.90$ & $17.43 \pm 39.45$ & $8.72 \pm 25.77$ \\
AFP $(\mathrm{ng} / \mathrm{mL})$ & $3.5 I \pm 2.03$ & $3.52 \pm 2.09$ & $2.49 \pm 1.97$ \\
CAI25 $(\mathrm{U} / \mathrm{mL})$ & $19.69 \pm 19.47$ & $22.40 \pm 21.80$ & $16.62 \pm 16.07$ \\
CAI99 $(\mathrm{U} / \mathrm{mL})$ & $47.51 \pm 114.82$ & $48.37 \pm 113.52$ & $46.54 \pm 117.08$ \\
\hline
\end{tabular}


level of CEA was $17.43 \pm 39.45 \mathrm{ng} / \mathrm{mL}$, AFP was $3.52 \pm 2.09$ $\mathrm{ng} / \mathrm{mL}$, CA125 was $22.40 \pm 21.80 \mathrm{U} / \mathrm{mL}$ and CA199 was $48.37 \pm 113.52 \mathrm{U} / \mathrm{mL}$ in the colon cancer group; CEA was $8.72 \pm 25.77 \mathrm{ng} / \mathrm{mL}$, AFP was $2.49 \pm 1.97 \mathrm{ng} / \mathrm{mL}$, CA125 was $16.62 \pm 16.07 \mathrm{U} / \mathrm{mL}$ and CA199 was 46.54 $\pm 117.08 \mathrm{U} / \mathrm{mL}$ in the rectal cancer group.

\section{Correlativity Between CTCs and Clinical Pathological Features}

The correlativity between CTC counts and clinical pathological features was investigated in this cohort. In detail, 72 cases were CTC-positive $(\mathrm{CTC}+)$ and 77 cases were CTC-negative (CTC-). The patients' general situation, tumor stage and tumor markers were compared between the two groups (Table 2). There was no significant difference between the groups in their general condition. However, there were significant statistical differences in tumor stage and tumor markers between the CTC+ and CTC - groups. There were eight cases with stage I, 16 cases with stage II, 36 cases with stage III and 12 cases with stage IV disease in the $\mathrm{CTC}+$ group; and 34 cases with stage I, 19 cases with stage II, 22 cases with stage III and two cases with stage IV disease in the CTC- group $(P=0.000)$. The measurement of serum biomarkers in the $\mathrm{CTC}+$ and $\mathrm{CTC}-$ groups indicated significantly increased expression in the CTC+ group (CEA: $19.04 \pm 44.43 \mathrm{ng} / \mathrm{mL}$

Table 2 Comparison Between CTCNegative and CTCPositive Groups

\begin{tabular}{|l|c|c|c|}
\hline & CTC+ $(\mathbf{n}=\mathbf{7 2})$ & CTC $-(\mathbf{n}=\mathbf{7 7})$ & $P$ \\
\hline Age (years) & $63.22 \pm 12.10$ & $63.16 \pm 12.10$ & 0.973 \\
Gender $(\mathrm{M} / \mathrm{F})$ & $47 / 25$ & $46 / 31$ & 0.486 \\
BMI $\left(\mathrm{kg} / \mathrm{m}^{2}\right)$ & $22.50 \pm 3.66$ & $22.70 \pm 3.11$ & 0.716 \\
\hline Stage I & 8 & 34 & 0.000 \\
Stage II & 16 & 19 & \\
Stage III & 36 & 22 & \\
Stage IV & 12 & 2 & \\
\hline CTC count/7.5 mL & $7.75 \pm 3.26$ & $1.94 \pm 0.92$ & 0.000 \\
\hline CEA (ng/mL) & $19.04 \pm 44.43$ & $8.00 \pm 18.26$ & 0.053 \\
AFP $(\mathrm{ng} / \mathrm{mL})$ & $3.95 \pm 2.01$ & $3.10 \pm 1.97$ & 0.010 \\
CAI25 (U/mL) & $27.97 \pm 24.17$ & $11.94 \pm 8.17$ & 0.000 \\
CAI99 (U/mL) & $62.15 \pm 117.85$ & $33.82 \pm 110.92$ & 0.133 \\
\hline Colon cancer & 41 & 38 & 0.353 \\
Rectal cancer & 31 & 39 & \\
Left hemicolon cancer & 39 & 46 & 0.492 \\
Right hemicolon cancer & 33 & 31 & \\
\hline
\end{tabular}

vs $8.00 \pm 18.26 \mathrm{ng} / \mathrm{mL}, P=0.053$; AFP: $3.95 \pm 2.01 \mathrm{ng} / \mathrm{mL}$ vs $3.10 \pm 1.97 \mathrm{ng} / \mathrm{mL}, P=0.010$; CA125: $27.97 \pm 24.17 \mathrm{U} / \mathrm{mL}$ vs $11.94 \pm 8.17 \mathrm{U} / \mathrm{mL}, P=0.000$; CA199: $62.15 \pm 117.85 \mathrm{U} / \mathrm{mL}$ vs $33.82 \pm 110.92 \mathrm{U} / \mathrm{mL}, P=0.133$ ) (Table 2 ).

According to these results, we discovered that the clinical pathological features of this CRC cohort are in close accordance with CTC counts. To analyze and evaluate CTCs in different CTC subgroups, we further investigated the CTCs counts in colon/rectum and left/ right CRC subgroups (Table 2). The results showed that there were 41 cases in the CTC + group and 38 cases in the CTC - group with colon cancer; and 31 cases in the CTC+ group and 39 cases in the CTC- group with rectal cancer $(P=0.353)$. In another subgroup, there were 39 cases in the $\mathrm{CTC}+$ group and 46 cases in the CTC - group with left hemicolon cancer; and 33 cases in the $\mathrm{CTC}+$ group and 31 cases in the $\mathrm{CTC}^{-}$group with right hemicolon cancer $(P=0.492)$.

\section{Correlativity Between CTC Counts and Clinical Pathological Features in Colon and Rectal Cancer}

Because of the discovery of a correlation between tumor stage and number of CTCs, the correlativity of CTC

Table 3 Comparison Between CTC-Negative and CTC-Positive Groups in Different Tumor Stages in Patients with Colon Cancer and Rectal Cancer

\begin{tabular}{|c|c|c|c|c|c|c|}
\hline & \multicolumn{2}{|c|}{$\begin{array}{l}\text { Colon Cancer } \\
\qquad(n=79)\end{array}$} & \multirow[t]{2}{*}{$P$} & \multicolumn{2}{|c|}{$\begin{array}{l}\text { Rectal Cancer } \\
\qquad(n=70)\end{array}$} & \multirow[t]{2}{*}{$P$} \\
\hline & $\begin{array}{l}\text { СTC- } \\
(0-3)\end{array}$ & CTC+ & & $\begin{array}{l}\text { СTC- } \\
(0-3)\end{array}$ & CTC+ & \\
\hline Stage I & 18 & 5 & 0.001 & 16 & 3 & 0.013 \\
\hline Stage II & 10 & 10 & & 9 & 6 & \\
\hline Stage III & 9 & 17 & & 13 & 19 & \\
\hline Stage IV & I & 9 & & I & 3 & \\
\hline TI & 10 & I & 0.021 & 9 & I & 0.001 \\
\hline T2 & 8 & 9 & & 12 & 3 & \\
\hline T3 & 9 & 13 & & 16 & 17 & \\
\hline $\mathrm{T} 4$ & II & 18 & & 2 & 10 & \\
\hline No & 28 & 18 & 0.014 & 25 & II & 0.035 \\
\hline $\mathrm{NI}$ & 9 & 16 & & 10 & 9 & \\
\hline N2 & 1 & 7 & & 4 & 11 & \\
\hline Mo & 37 & 33 & 0.018 & 38 & 28 & 0.203 \\
\hline MI & I & 8 & & I & 3 & \\
\hline
\end{tabular}


counts and clinicopathological features was analyzed further in the colon and rectum subgroups (Table 3).

In the colon cancer group, there were $38 \mathrm{CTC}-$ cases, comprising 18 cases with stage I, 10 cases with stage II, nine cases with stage III and one case with stage IV; and $41 \mathrm{CTC}+$ cases, comprising five cases with stage I, 10 cases with stage II, 17 cases with stage III and nine cases with stage IV $(P=0.001)$. In the rectal cancer group, there were $39 \mathrm{CTC}-$ cases, comprising 16 cases with stage I, nine cases with stage II, 13 cases with stage III and one case with stage IV; and $31 \mathrm{CTC}+$ cases, comprising three cases with stage I, six cases with stage II, 19 cases with stage III and three cases with stage IV $(P=0.013)$.

Besides, the statistics of patients with colon cancer suggested that the positive rate of CTCs was $21.74 \%$ in stage I, $50.00 \%$ in stage II, $65.38 \%$ in stage III and $90.00 \%$ in stage IV $(P=0.405)$; in the cases with rectal cancer, the positive rate of CTCs was $15.79 \%$ in stage I, $40.00 \%$ in stage II, $69.38 \%$ in stage III and $75.00 \%$ in stage IV ( $P=0.261$ ). The results showed that CTC counts increased in colon cancer along with the increase in tumor stage in colon cancer and rectal cancer.

Moreover, CTC-positive and -negative numbers in different TNM stages were compared separately in the colon cancer group and rectal cancer group (Table 3 ). In the colon cancer group, there were $38 \mathrm{CTC}-$ cases, comprising 10 cases with stage $\mathrm{T} 1$, eight cases with stage $\mathrm{T} 2$, nine cases with stage $\mathrm{T} 3$ and 11 cases with stage $\mathrm{T} 4$; and $41 \mathrm{CTC}+$ cases, comprising one case with stage $\mathrm{T} 1$, nine cases with stage T2, 13 cases with stage $\mathrm{T} 3$ and 18 cases with stage $\mathrm{T} 4$ $(P=0.021)$. In the rectal cancer group, there were $39 \mathrm{CTC}-$ cases, comprising nine cases with stage T1, 12 cases with stage T2, 16 cases with stage T3 and two cases with stage T4; and $31 \mathrm{CTC}+$ cases, comprising one case with stage T1, three cases with stage T2, 17 cases with stage T3 and 10 cases with stage $\mathrm{T} 4(P=0.001)$. In the colon cancer group, there were $38 \mathrm{CTC}-$ cases, comprising 28 cases with stage $\mathrm{N} 0$, nine cases with stage $\mathrm{N} 1$ and one case with stage N2; and $41 \mathrm{CTC}+$ cases, comprising 18 cases with stage N0, 16 cases with stage $\mathrm{N} 1$ and seven cases with stage N2 $(P=0.014)$. In the rectal cancer group, there were 39 CTC - cases, comprising 25 cases with stage N0, 10 cases with stage $\mathrm{N} 1$ and four cases with stage $\mathrm{N} 2$; and $31 \mathrm{CTC}+$ cases, comprising 11 cases with stage N0, nine cases with stage $\mathrm{N} 1$ and 11 cases with stage $\mathrm{N} 2(P=0.035)$. In the colon cancer group, there were $38 \mathrm{CTC}-$ cases, comprising 37 cases with stage M0 and one case with stage M1; and 41 $\mathrm{CTC}+$ cases, comprising 33 cases with stage M0 and eight cases with stage $\mathrm{M} 1(P=0.018)$. In the rectal cancer group, there were $39 \mathrm{CTC}-$ cases, comprising 38 cases with stage M0 and one case with stage M1; and $31 \mathrm{CTC}+$ cases, comprising 28 cases with stage M0 and three cases with stage M1 $(P=0.203)$.

The statistics of patients with colon cancer suggest that the positive rate of CTCs was $9.09 \%$ in stage T1, 52.94\% in stage T2, 59.09\% in stage T3 and $62.07 \%$ in stage T4 in the colon cancer group $(P=0.261)$; and $15.79 \%$ in stage $\mathrm{T} 1,40.00 \%$ in stage $\mathrm{T} 2,59.38 \%$ in stage $\mathrm{T} 3$ and $75.00 \%$ in stage $\mathrm{T} 4$ in the rectal cancer group $(P=0.261)$. In $\mathrm{N}$ staging, the positive rate of CTCs was $39.13 \%$ in stage N0, $64.00 \%$ in stage $\mathrm{N} 1$ and $87.5 \%$ in stage $\mathrm{N} 2$ in the colon cancer group $(P=0.223)$; and $30.56 \%$ in stage $\mathrm{N} 0,47.37 \%$ in stage N1 and $73.33 \%$ in stage N2 in the rectal cancer group ( $P=0.223)$. In $\mathrm{M}$ staging, the positive rate of CTCs was $47.14 \%$ in stage M0 and $88.89 \%$ in stage M1 in the colon cancer group ( $P=0.157)$; and $42.42 \%$ in stage M0 and $75.00 \%$ in stage M1 in the rectal cancer group $(P=0.157)$.

According to these results, the following conclusions can be summarized. With the increase in tumor stage, the positive rate of CTCs showed a rising trend in colon cancer and rectal cancer, especially in the colon cancer group. A similar phenomenon could be found between the CTC-positive rate and different $\mathrm{T}, \mathrm{N}, \mathrm{M}$ stages. These results show that the positive rate of CTCs and number of CTCs may be related to the depth of infiltration and lymphatic metastasis.

\section{Correlativity Between CTC Counts and Clinical Pathological Features in Left Hemicolon Cancer and Right Hemicolon Cancer}

In order to confirm the correlativity between CTCs and histoembryological differences, the patients were divided according to another tumor location, into the left hemicolon cancer group and right hemicolon cancer group (Table 4).

In the left hemicolon cancer group, there were 46 CTCcases, comprising 21 cases with stage I, 10 cases with stage II, 14 cases with stage III and one case with stage IV; and 39 CTC + cases, comprising three cases with stage I, eight cases with stage II, 24 cases with stage III and four cases with stage IV $(P=0.001)$. In the right hemicolon cancer group, there were $31 \mathrm{CTC}-$ cases, comprising 13 cases with stage I, nine cases with stage II, eight cases with stage III and one case with stage IV; and $33 \mathrm{CTC}+$ cases, comprising five cases 
Table 4 Comparison Between CTC-Negative and CTC-Positive Groups in Different Stages in Patients with Left- and Right-Side CRC

\begin{tabular}{|c|c|c|c|c|c|c|}
\hline & \multicolumn{2}{|c|}{$\begin{array}{l}\text { Left-Side CRC } \\
\qquad(n=85)\end{array}$} & \multirow[t]{2}{*}{$P$} & \multicolumn{2}{|c|}{$\begin{array}{c}\text { Right-Side CRC } \\
(n=64)\end{array}$} & \multirow[t]{2}{*}{$P$} \\
\hline & $\begin{array}{l}\text { CTC- } \\
(0-3)\end{array}$ & CTC+ & & $\begin{array}{l}\text { CTC- } \\
(0-3)\end{array}$ & CTC+ & \\
\hline Stage I & 21 & 3 & 0.001 & 13 & 5 & 0.020 \\
\hline Stage II & 10 & 8 & & 9 & 8 & \\
\hline Stage III & 14 & 24 & & 8 & 12 & \\
\hline Stage IV & I & 4 & & 1 & 8 & \\
\hline TI & II & I & 0.000 & 8 & I & 0.059 \\
\hline $\mathrm{T} 2$ & 15 & 4 & & 5 & 8 & \\
\hline T3 & 18 & 18 & & 7 & 12 & \\
\hline T4 & 2 & 16 & & 11 & 12 & \\
\hline No & 31 & 14 & 0.005 & 22 & 15 & 0.077 \\
\hline $\mathrm{NI}$ & 11 & 12 & & 8 & 13 & \\
\hline N2 & 4 & 13 & & 1 & 5 & \\
\hline MO & 45 & 36 & 0.231 & 30 & 25 & 0.016 \\
\hline MI & I & 3 & & I & 8 & \\
\hline
\end{tabular}

with stage I, eight cases with stage II, 12 cases with stage III and eight cases with stage IV $(P=0.020)$.

The statistics of patients with left hemicolon cancer suggest that the positive rate of CTCs was $12.50 \%$ in stage I, $44.44 \%$ in stage II, $63.16 \%$ in stage III and $80.00 \%$ in stage IV $(P=0.261)$; in the cases with right hemicolon cancer, the positive rates of CTCs were $27.78 \%$ in stage I, $47.06 \%$ in stage II, $60.00 \%$ in stage III and $88.89 \%$ in stage IV $(P=0.261)$. The results showed that the positive rate of CTCs increased in both groups along with the increase in tumor stage.

Comparing the two groups separately, in the left hemicolon cancer group, there were $46 \mathrm{CTC}-$ cases, comprising 11 cases with stage T1, 15 cases with stage T2, 18 cases with stage $\mathrm{T} 3$ and two cases with stage $\mathrm{T} 4$; and $39 \mathrm{CTC}+$ cases, comprising one case with stage $\mathrm{T} 1$, four cases with stage $\mathrm{T} 2$, 18 cases with stage T3 and 16 cases with stage T4 $(P=0.000)$. In the right hemicolon cancer group, there were $31 \mathrm{CTC}-$ cases, comprising eight cases with stage T1, five cases with stage $\mathrm{T} 2$, seven cases with stage $\mathrm{T} 3$ and 11 cases with stage T4; and 33 CTC + cases, comprising one case with stage T1, eight cases with stage T2, 12 cases with stage T3 and 12 cases with stage $\mathrm{T} 4(P=0.059)$. In the left hemicolon cancer group, there were $46 \mathrm{CTC}-$ cases, comprising 31 cases with stage N0, 11 cases with stage $\mathrm{N} 1$ and four cases with stage $\mathrm{N} 2$; and $39 \mathrm{CTC}+$ cases, comprising 14 cases with stage N0,
12 cases with stage $\mathrm{N} 1$ and 13 cases with stage $\mathrm{N} 2$ $(P=0.005)$. In the right hemicolon cancer group, there were 31 CTC cases, comprising 22 cases with stage N0, eight cases with stage $\mathrm{N} 1$ and one case with stage $\mathrm{N} 2$; and 33 cases with $\mathrm{CTC}+$ cases, comprising 15 cases with stage N0, 13 cases with stage $\mathrm{N} 1$ and five cases with stage N2 $(P=0.077)$. In the left hemicolon cancer group, there were $46 \mathrm{CTC}$ cases, comprising 45 cases with stage M0 and one case with stage $\mathrm{M} 1$; and 39 cases with $\mathrm{CTC}+$ cases, comprising 36 cases with stage $\mathrm{M} 0$ and three cases with stage M1 $(P=0.231)$. In the right hemicolon cancer group, there were $31 \mathrm{CTC}-$ cases, comprising 30 cases with stage $\mathrm{M} 0$ and one case with stage M1; there were 33 cases with CTC + cases, comprising 25 cases with stage $\mathrm{M} 0$ and eight cases with stage M1 $(P=0.016)$.

The statistics of patients in the left and right hemicolon groups suggest that the positive rate of CTCs was $15.74 \%$ in stage $\mathrm{T} 1,21.05 \%$ in stage $\mathrm{T} 2,50.00 \%$ in stage T3 and $88.89 \%$ in stage T4 in the left hemicolon cancer group $(P=0.405)$; and $11.11 \%$ in stage $\mathrm{T} 1$, $61.54 \%$ in stage $\mathrm{T} 2,63.16 \%$ in stage $\mathrm{T} 3$ and $52.17 \%$ in stage T4 in the right hemicolon cancer group $(P=0.261)$. In $\mathrm{N}$ staging, the positive rate of CTCs was $31.11 \%$ in stage N0,52.17\% in stage N1 and $76.47 \%$ in stage N2 in the left hemicolon cancer group ( $P=0.223$ ); and $40.54 \%$ in stage $\mathrm{N} 0,61.90 \%$ in stage $\mathrm{N} 1$ and $83.33 \%$ in stage $\mathrm{N} 2$ in the right hemicolon cancer group $(P=0.223)$. In $M$ staging, the positive rate of CTCs was $39.56 \%$ in stage M0 and $75.00 \%$ in stage M1 in the left hemicolon cancer group ( $P=0.157)$; and $45.45 \%$ in stage M0 and $88.89 \%$ in stage $\mathrm{M} 1$ in the rectal cancer group $(P=0.157)$.

According to these results, the following conclusions can be summarized. With increasing in tumor stage, the positive rate of CTCs showed a rising trend in both left and right hemicolon cancer. Between the $\mathrm{CTC}+$ and $\mathrm{CTC}-$ groups, differences were found in $\mathrm{T}$ stage and $\mathrm{N}$ stage, especially with left hemicolon cancer. These results show that the positive rate of CTCs may be related to the depth of infiltration and lymphatic metastasis, especially in the left hemicolon cancer group.

\section{Correlativity Between CTCs and Prognosis of Survival}

In this study, the average overall survival (OS) was 23.66 \pm 5.20 months in the CTC- group and $23.52 \pm 5.20$ months in the $\mathrm{CTC}+$ group $(P=0.874)$. 
There were 29 cases with tumor recurrence and 27 cases with tumor-related death in the $\mathrm{CTC}+$ group; and nine cases with tumor recurrence and six cases with tumorrelated death in the CTC- group.

The overall survival and progression-free survival curves of the patients with CRC in this trial were established to evaluate the compariative risk between the CTC- and CTC + groups (Figures 1 and 2). The results showed that there was a steep gradient in survival during the follow-up period, particularly in the CTC + group $(P=0.000,0.000)$. On the risk assessment curves, the change escalates more rapidly in the CTC+ group.
The change in survival and risk functions may be related with to the tumor stage and the depth of tumor invasion. Therefore, we further analyzed the correlativity between CTCs and different tumor stage and different tumor T staging (stages I-III, stages T2-4) (Figures 3 and 4). The results showed that with the increase in tumor stage and $\mathrm{T}$ stage, changes in survival curve and risk curve escalate more rapidly in the $\mathrm{CTC}+$ group. The change in survival and risk functions may also be related to postoperative therapy. As the patients in the CTCpositive group showed relatively advanced tumor stage, which had received regular postoperative treatment
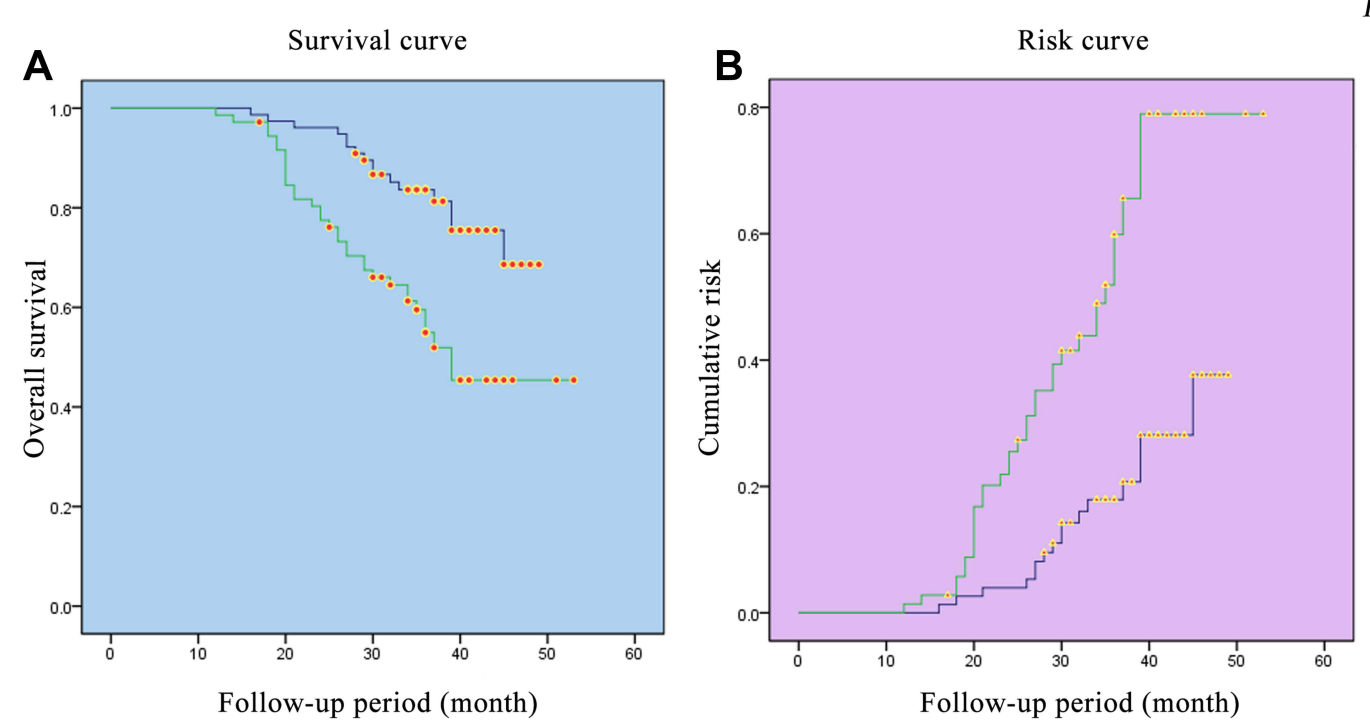

$P=0.000$

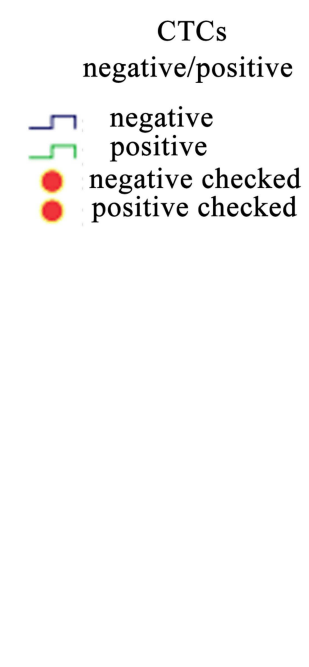

Figure I Survival curve and risk curve in the CRC cohort. (A) Survival curve in the CRC cohort. (B) Risk curve in the CRC cohort.
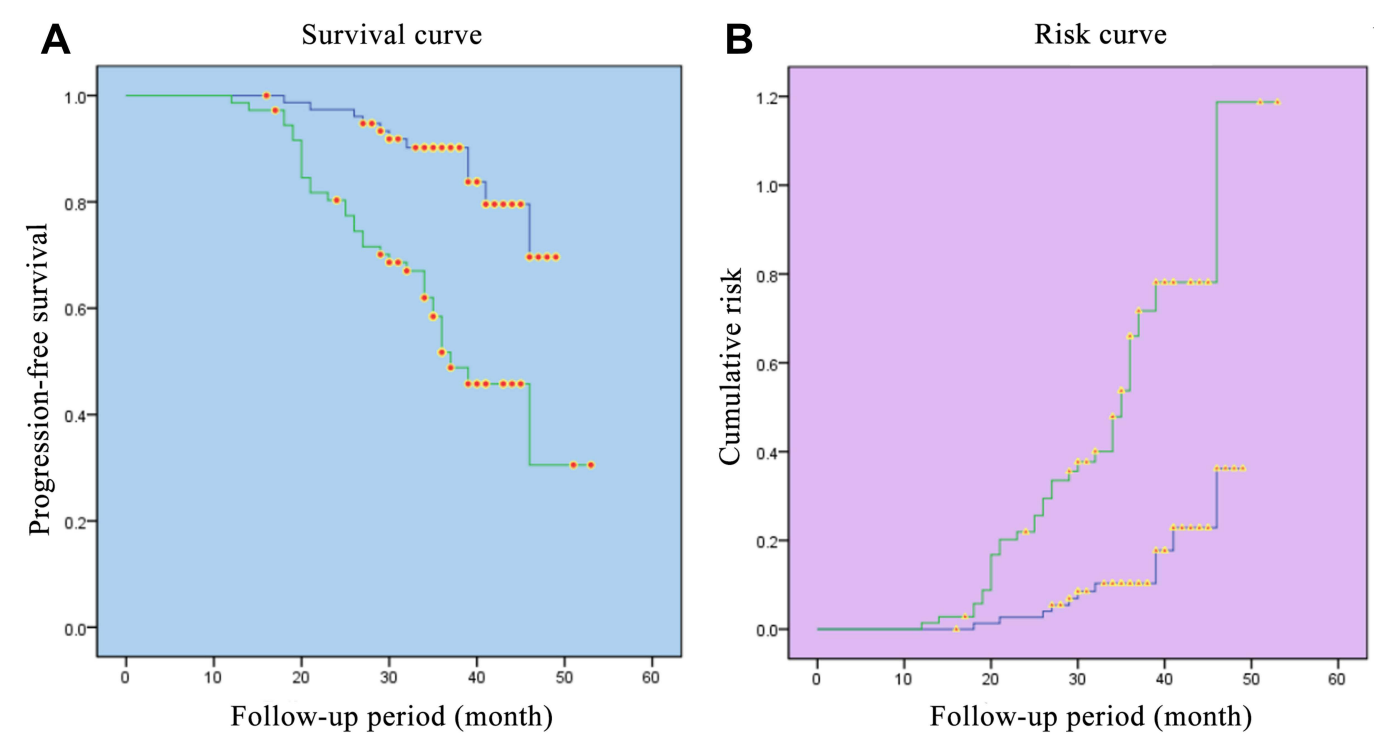

$P=0.000$

CTCs negative/positive

$\neg$ negative $\neg$ positive negative checked positive checked

Figure 2 Progression-free survival curve and risk curve of colorectal patients. (A) Progression-free survival curve of colorectal patients. (B) Risk curve of colorectal patients. 

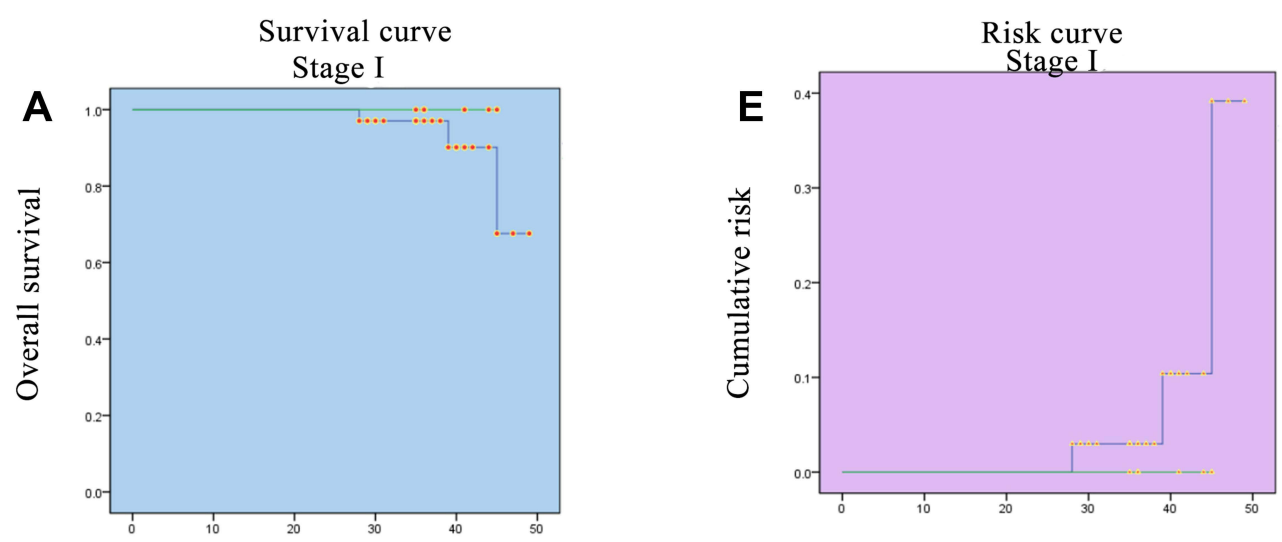

CTCs

negative/positive

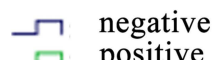

ositive

negative checked positive checked
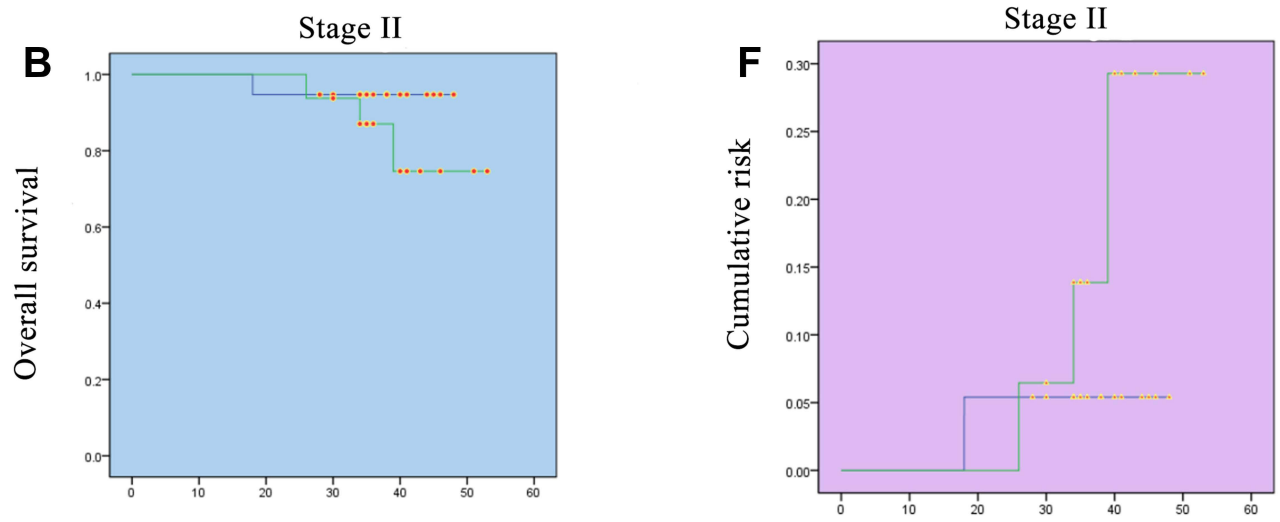

CTCs

negative/positive

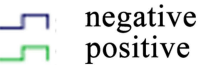

$\neg$ positive

- negative checked

positive checked
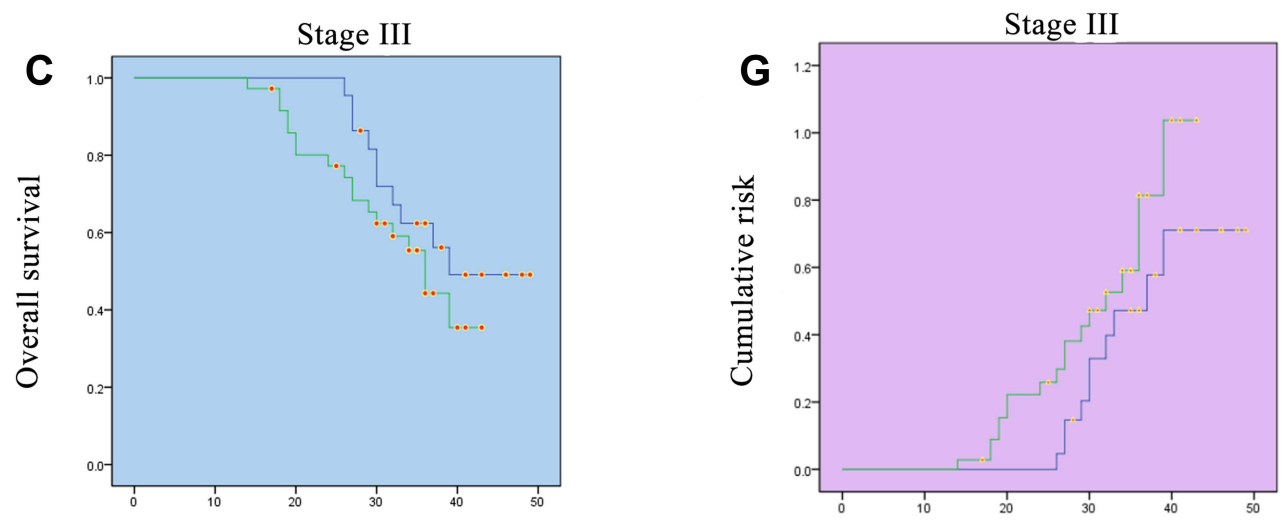

CTCs

negative/positive

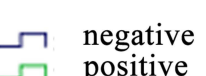

negative checked positive checked
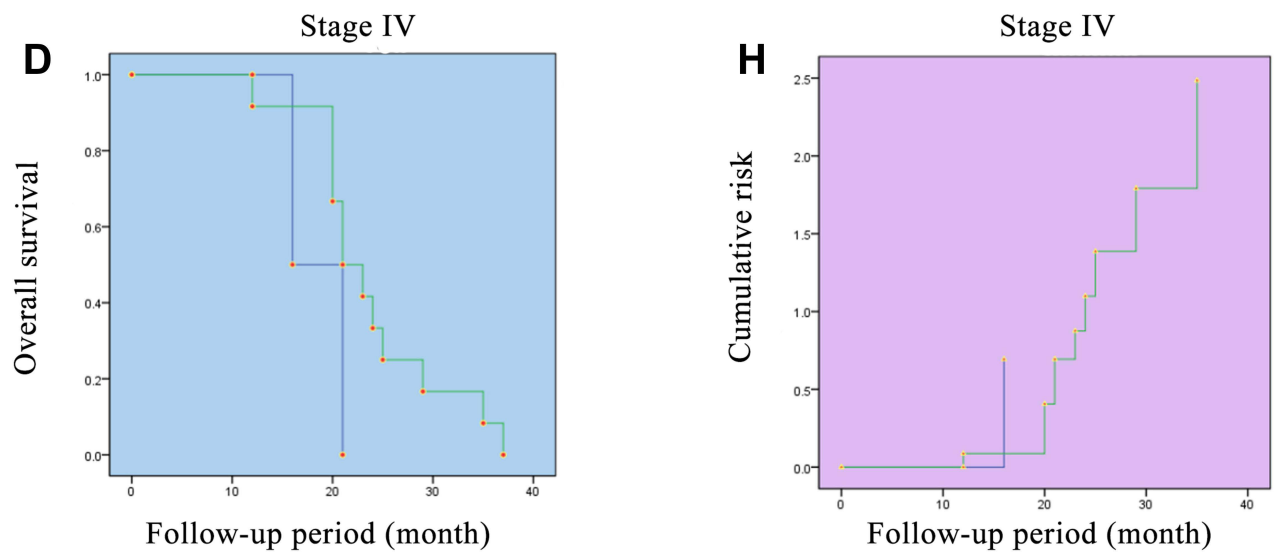

CTCs negative/positive

$\neg$ negative

$\neg$ positive

- negative checked positive checked

Figure 3 Survival curves and risk curves of colorectal patients in different stages. (A) Survival curve of colorectal patients in stage I. (B) Survival curve of colorectal patients in stage II. (C) Survival curve of colorectal patients in stage III. (D) Survival curve of colorectal patients in stage IV. (E) Risk curve of colorectal patients in stage I. (F) Risk curve of colorectal patients in stage II. (G) Risk curve of colorectal patients in stage III. (H) Risk curve of colorectal patients in stage IV. 

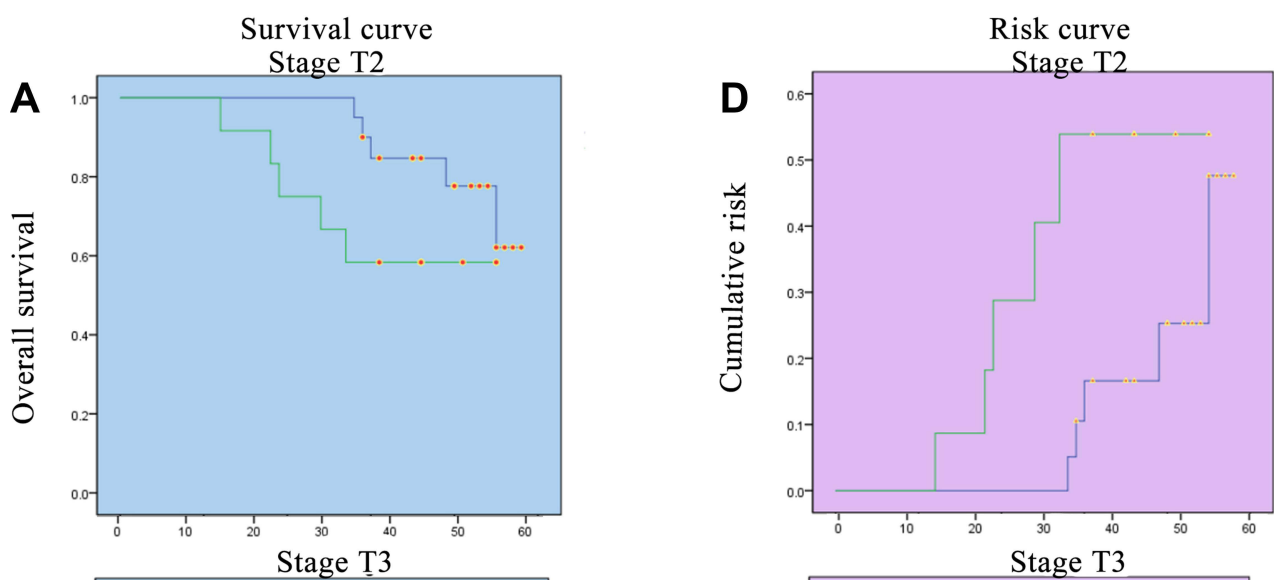

CTCs

negative/positive

$\neg$ negative

$\neg$ positive

- negative checked

- positive checked
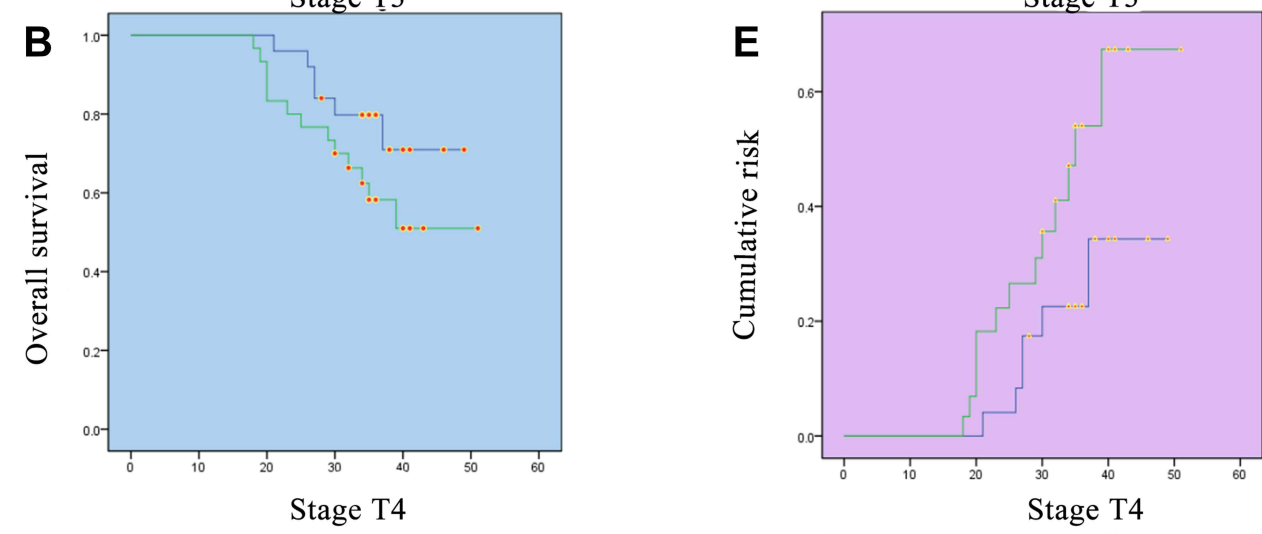

CTCs negative/positive

$\neg$ negative

$\neg$ positive

- negative checked

positive checked
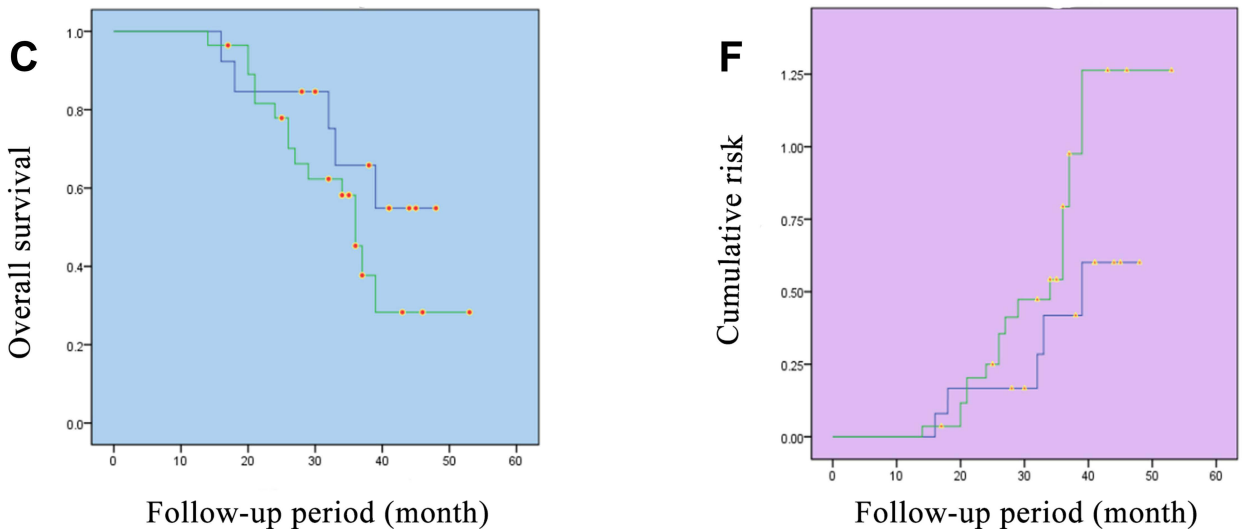

CTCs negative/positive negative positive negative checked positive checked

Figure 4 Survival curves and risk curves of colorectal patients in different T stages. (A) Survival curve of colorectal patients in stage T2. (B) Survival curve of colorectal patients in stage T3. (C) Survival curve of colorectal patients in stage T4. (D) Risk curve of colorectal patients in stage T2. (E) Risk curve of colorectal patients in stage T3. (F) Risk curve of colorectal patients in stage T4.

according to the NCCN guideline, they may have achieved some degree of tumor control. LLower survival rates could also be discovered in patients with advanced CRC.

\section{Discussion}

$\mathrm{CRC}$ is one of the most common malignant tumors in China. It threatens people's health and influences the quality of people's life due to its rising incidence in recent years. ${ }^{4}$ Not all digestive cancers can be diagnosed in a timely manner, although some tumor markers (eg, CEA, CA125,
CA199, AFP) are available to assist in the diagnosis. As the elevation of tumor markers is not always seen in early cases, it is difficult to make an early diagnosis and estimate the long-term prognosis of cancer patients. The major causes of death from CRC are tumor recurrence and distant metastasis. Even in patients diagnosed with advanced stage cancer or with metastasis, these tumor markers may not be elevated at all. Therefore, exploring the effective index to estimate long-term prognosis for all stages of CTC is one of the main focuses of research. 
The mechanism of tumor recurrence is complex and diverse. We think that CTCs may play an important part in tumor recurrence and distant metastasis. CTCs are exfoliative cells of the primary tumor or metastatic tumor, which can enter the bloodstream. ${ }^{19}$ This kind of cell could be undetected by the immune system, ultimately leading to distant metastases. ${ }^{20,21}$ Therefore, CTCs could be a useful tumor marker for anticipating the survival prognosis and evaluation for advanced stage CRC. ${ }^{22}$ In this study, significant differences were found between different tumor stages in CRC, and the colon cancer group and right hemicolon group showed much higher sensitivity in the positive rate of CTCs.

Patients with CRC had their CTCs examined, and data on prognosis were collected and analyzed in this study. The results showed that the recurrence rate of the tumor was higher in the CTC+ group, which means that tumor recurrence has a close interrelation with the number of CTCs in the peripheral blood of patients. The results indicated that dynamic monitoring of CTCs could be a valuable clinical tool for prognostic evaluation. The metastasis rate has been reported to be up to $24 \%$ in the CTC-positive group in the case of colon cancer. ${ }^{23}$ Moreover, it was accompanied by elevation of CTCs in cases of lung cancer, breast cancer, urothelial carcinoma and gastric cancer. ${ }^{24-28}$ Since 2004, a number of CTCs has been approved by the FDA to evaluate the ecological prognosis and outcome. In the current study, it was shown that the higher the number of CTCs in the serum, the worse the prognosis for the patient. During chemotherapy, a significantly reduced number of CTCs may be an indicator of favorable prognosis. ${ }^{28}$ In this study, the results showed that the sensitivity of CTCs may be related to the histoembryological location of the tumor and the depth of tumor invasion.

The results of other researchers have shown that the change in the number of CTCs could have a detectable effect in assessing the effectiveness of radical resection. ${ }^{29,30}$ The liver is the most common site affected by gastrointestinal tumor metastasis. It is reported that the number of CTCs in serum could be increased to seven times the postoperative levels by radiofrequency ablation of metastases. ${ }^{31}$ This result indicates that damaging tumor may cause an elevation of the local recurrence rate by hematogenous metastasis. With the advent of total mesorectal excision (TME) and complete mesocolic excision (CME) in the surgical field, the anatomy of the mesenterium is receiving much more attention and could dramatically improve the prognosis of patients. ${ }^{32}$ Based on these experimental results, the number of CTCs could be an evaluative criterion to assess the quality of surgery. In clinical practice, the surgeon could also compare the preoperative and postoperative numbers of CTCs in serum to assess the pathological evaluation and estimate the risk of hematogenous metastasis. The patients had no other risk factors, but an increased level of CTCs on two successive occasions could be a valuable finding. Because the focus of this study was the correlativity between the sensitivity of CTCs and clinicopathological features, no further analysis was carried out to verify the correlativity between CTC counts and surgical quality. Large-sample, multicenter, double-blind, randomized, controlled clinical trials are still needed to study the clinical applied value of CTCs for surgical evaluation

The promise of personalized, precision medicine has become a hot medical topic in recent years. Cancer molecular targeting therapy has limited or non-existent side effects on normal cells of the body, unlike traditional chemotherapy. However, the target gene expression is frequently negative in the primary lesion, leading to difficulties in treatment in cases with metastasis. Research has shown that estrogen receptors were negative in $40 \%$ cases of breast cancer in the primary lesion or metastatic lesion, but positive in detecting CTCs; $23 \%$ cases of breast cancer were HER-2 negative but CTCs were positive. ${ }^{33}$ These results showed that the applied value of CTCs not only is limited within tumor screening, diagnosis and prognositic evaluation, but also has become a key point for effective individual targeted therapy.

Tumor recurrence is one of the leading causes of death in cases of digestive cancer, and CTCs are considered to be a key factor in hematogenous metastases. CTCs could not only be used to assist in screening patients at high risk, but also be useful in the development of accurate treatment by providing treatment evidence. ${ }^{34}$ In the past, the diagnosis of cancer largely depended on surgical biopsy, endoscopic biopsy and interventional biopsy to guide therapeutic strategies. These kinds of examination are invasive procedures that may be difficult to carry out if patients suffer in the examination. Detecting CTCs in the serum would be a convenient and sensitive test with the advantages of convenient collection, less trauma and being acceptable to patients. Until now, there have been no studies demonstrating the relationship between the threshold number of CTCs and tumor recurrence or metastasis. In this cohort study, significant differences could be found 
after analyzing the survival curves and risk curves for different $\mathrm{T}$ stages in the CTC-negative and CTC-positive groups. Further research with a larger sample is required to investigate the clinical value of CTCs for clinical prognosis and assessment.

\section{Conclusion}

By combining the analyses, the following conclusions can be made. 1) There were significant differences between different tumor stages in patients with CRC; 2) compared with the rectal cancer group, the colon cancer group showed much higher sensitivity in the positive rate of CTCs; 3) compared with the right hemicolon group, the left hemicolon group showed much higher sensitivity in the positive rate of CTCs; 4) the sensitivity of CTCs may be related to the histoembryological location of the tumor, lymphatic metastasis and the depth of tumor invasion; 5) an overall survival benefit was found in the CTC- group; and 6) with increasing tumor stage and T stage, changes in the survival curve and risk curve escalated more rapidly in the CTC+ group.

In summary, it was confirmed that in the left hemicolon cancer group, much higher coincidence rate could be found on CTC-positive rate and clinicopathological features, than in the right hemicolon cancer group The sensitivity of CTCs may be related to the histoembryological location of the tumor, lymphatic metastasis and the depth of infiltration. Monitoring CTCs may have value in evaluating clinical staging and estimating clinical prognosis.

\section{Author Contributions}

All authors made a significant contribution to the work reported, whether that is in the conception, study design, execution, acquisition of data, analysis and interpretation, or in all these areas; took part in drafting, revising or critically reviewing the article; gave final approval of the version to be published; have agreed on the journal to which the article has been submitted; and agree to be accountable for all aspects of the work.

\section{Funding}

There is no funding to report.

\section{Disclosure}

The authors declared no conflicts of interest for this work.

\section{References}

1. Torre LA, Bray F, Siegel RL, Ferlay J, Lortet-Tieulent J, Jemal A. Global cancer statistics, 2012. CA Cancer J Clin. 2015.

2. Glynne-Jones R, Wyrwicz L, Tiret E, et al. Rectal cancer: ESMO Clinical Practice Guidelines for diagnosis, treatment and follow-up. Ann Oncol. 2018;1(29):22-40.

3. Benson AB 3rd, Venook AP, Al-Hawary MM, et al. Rectal Cancer, Version 2.2018, NCCN Clinical Practice Guidelines in Oncology. 2018;16(7):874-901.

4. Chinese Society of Clinical Oncology. Guidelines for the diagnosis and treatment of colorectal cancer in China (Version 2017). 2017.

5. Chen WQ, Zheng RS, He J, et al. Cancer incidence and mortality in China, 2013. Cancer Letters. 2017;10:63-71.

6. Zhong W, Yu Z, Zhan J, et al. Association of serum levels of CEA, CA199, CA125, CYFRA21-1 and CA72-4 and disease characteristics in colorectal cancer. Pathol Oncol Res. 2015;21(1):83-95.

7. He B, Zhang HQ, Xiong SP, et al. Changing patterns of serum CEA and CA199 for evaluating the response to first-line chemotherapy in patients with advanced gastric adenocarcinoma. Asian Pac J Cancer Prev. 2015;16(8):3111-3116.

8. Guo J, Chen S, Li S, et al. A novel classifier based on three preoperative tumor markers predicting the cancer-specific survival of gastric cancer (CEA, CA19-9 and CA72-4). Oncotarget. 2017;9 (4):4814-4822.

9. Husemann Y, Geigl JB, Schubert F, et al. Systemic spread is an early step in breast cancer. Cancer Cell. 2008;13(1):58-68.

10. Arigami T, Uneosono Y, Hirata M, et al. B7-H3 expression in gastric cancer: a novel molecular blood marker for detecting circulating tumor cell. Cancer Sci. 2011;102(5):1019-1024.

11. Cao W, Yang W, Li H, et al. Using detection of survivin-expressing circulating tumor cells in peripheral blood to predict tumor recurrence following curative resection of gastric cancer. J Surg Oncol. 2011;103(2):110-115.

12. Hayes DF, Cristofanilli M, Budd GT, et al. Circulating tumor cells at each follow-up time point during therapy of metastatic breast cancer patients predict progression-free and overall survival. Clin Cancer Res. 2006;12:4218-4224.

13. Ennis RD, Katz AE, de Vries GM, et al. Detection of circulating prostate carcinoma cells via an enhanced reverse transcriptasepolymerase chain reaction assay in patients with early stage prostate carcinoma independence from other pretreatment characteristics. Cancer. 1997;79:2402-2408.

14. de Bono JS, Scher HI, Montgomery RB, et al. Circulating tumor cells predict survival benefit from treatment in metastatic castration-resistant prostate cancer. Clin Cancer Res. 2008;14:6302-6309.

15. Riethdorf S, Muller V, Zgabg L, et al. Detection and HER2 expression of circulating tumor cells: prospective monitoring in breast cancer patients treated in the neoadjuvant GeparQuattro trial. Clin Cancer Res. 2010;16(9):2634-2645.

16. Lianidou ES, Markou A. Circulating tumor cells in breast cancer: detection systems, molecular characterization, and future challenges. Clin Chem. 2011;57(9):1242-1255.

17. Van Cutsem E, Cervantes A, Adam R, et al. ESMO consensus guidelines for the management of patients with metastatic colorectal cancer. Ann Oncol. 2016;27(8):1386-1422.

18. Yoshino T, Arnold D, Taniguchi H, et al. Pan-Asian adapted ESMO consensus guidelines for the management of patients with metastatic colorectal cancer: a JSMO-ESMO initiative endorsed by CSCO, KACO, MOS, SSO and TOS. Ann Oncol. 2018;29(1):44-70.

19. Elshimali YI, Grody WW. The clinical significance of circulating tumor cells in the peripheral blood. Diagn Mol Pathol. 2006;15 (4):187-194.

20. Qiao YY. Circulating tumor cells and the correlation with metastasis and recurrence of tumor. J Modern Oncol. 2011(19):1877-1880. 
21. Paget JA, Restall IJ, Daneshmand M, et al. Repression of cancer cell senescence by PKCiota. Oncogene. 2012;31(31):3584-3596.

22. Iinuma $\mathrm{H}$, Watanabe $\mathrm{T}$, Mimori $\mathrm{K}$, et al. Clinical significance of circulating tumor cells, including cancer stem-like cells, in peripheral blood for recurrence and prognosis in patients with Dukes' stage B and C colorectal cancer. J Clin Oncol. 2011;29(12):1547-1555.

23. Soster M, Juris R, Bonacchi S, et al. Targeted dual-color silica nanoparticles provide univocal identification of micmmetastases in preelinieal models of colorectal cancel. Int J Nanomedicine. 2012;7:4797.

24. Krebs MG, Sloane R, Priest L, et al. Evaluation and prognostic significance of circulating tumor cells in patients with non-smallcell lung cancer. J Clin Oncol. 2011;29(12):1556-1563.

25. Msaouel P, Koutsilieris M. Diagnostic value of circulating tumor cell detection in bladder and urothelial cancer: systematic review and meta-analysis. BMC Cancer. 2011;11:336.

26. Btilke E, Orth K, Gerber PA, et al. Gene expression of circulating turnout cells and its correlation witll tumor stage in breast cancer patients. Eur J Med Res. 2009;14(8):359-363.

27. Rink M, Chun FK, Dahlem R, et al. Prognostic role and HER2 expression of circulating tumor cells in peripheral blood of patients prior to radical cystectomy: a prospective study. Eur Urol. 2012;61(4):810-817.

28. Matsusaka S, Chin K, Ogura M, et al. Circulating tumor cells as a surrogate marker for determining response to chemotherapy in patients with advanced gastric cancer. Cancer Sci. 2010;101 (4):1067-1071.
29. Sun YF, Xu Y, Yang XR, et al. Circulating stem cell- like epithelial cell adhesion molecule-positive tumor cells indicate poor prognosis of hepatocellular carcinoma after curative resection. Hepatology. 2013;57(4):1458-1468.

30. Jatana KR, Balasubramanian P, Lang JC, et al. Significance of circulating tumor cells in patients with squamous cell carcinoma of the head and neck: initial results. Arch Otolaryngol Head Neck Surg. 2010;136(12):1274-1279.

31. Papavasiliou P, Fisher T, Kuhn J, et al. Circulating tumor cells in patients undergoing surgery for hepatic metastases from colorectal cancer. Proc (Bayl Univ Med Cent). 2010;23(1):11-14.

32. Ostenfeld EB, Erichsen R, Iversen LH, et al. of patients with colon and rectal cancer in central and northern Denmark, 1998-2009. Clin Epidemiol. 2011;3(Suppl 1):S27-34.

33. Somlo G, IJau SK, Frankel P, et al. Multipie biomarker expression on circulating tnlnor cells in comparison to tumor tissues from primary and metastatic sites in patients with locally advanced inflammatory, and stage IV breast cance using a novel detection technology. Breast Cancer Res Treat. 2011;128(1):155-163.

34. Vacante M, Ciuni R, Basile F, et al. The liquid biopsy in the management of colorectal cancer: an overview. Biomedicines. 2020;8(9):308.

\section{Publish your work in this journal}

Cancer Management and Research is an international, peer-reviewed open access journal focusing on cancer research and the optimal use of preventative and integrated treatment interventions to achieve improved outcomes, enhanced survival and quality of life for the cancer patient.
The manuscript management system is completely online and includes a very quick and fair peer-review system, which is all easy to use. Visit http://www.dovepress.com/testimonials.php to read real quotes from published authors. 